\title{
No período pós-parto o uso dos contraceptivos orais combinados é tão seguro quanto o uso dos progestativos orais?
}

Luís Filipe Cavadas*

\section{RESUMO}

Introdução: A Contracepção Hormonal $(\mathrm{CH})$ no período pós-parto é um problema de saúde pública de grande importância, com implicações para a mulher e para a criança que é amamentada.

Objectivos: Determinar a segurança dos Contraceptivos Orais Combinados (COCs) e dos Progestativos Orais (POs) para a mulher no período pós-parto e para a criança que é amamentada.

Métodos: Foi realizada uma pesquisa nas bases de dados MEDLINE, Guidelines Finder, National Guideline Clearinghouse, Canadian Medical Association Infobase, The Cochrane Library, DARE, Bandolier, TRIP, InfoPoems e Sociedades Científicas utilizando os termos MeSH: Contraceptives, Oral; Safety; Postpartum Period; e o termo Postpartum. Limitou-se a pesquisa a artigos publicados desde Janeiro de 2000 até Abril de 2008, em Inglês, Português, Francês e Castelhano. Para a comparação de resultados, foram usadas as categorias dos Critérios de Elegibilidade Médica da Organização Mundial de Saúde (CEMOMS). Para avaliar o nível de evidência, foi utilizada a escala de Strength of Recommendation Taxonomy (SORT) da American Family Physician.

Resultados: Duas meta-análises, uma revisão sistemática, duas revisões baseadas na evidência e cinco normas de orientação clínica preencheram os critérios de inclusão. Segurança para a mulher: Existe uma preocupação teórica acerca da associação do uso dos COCs até às três semanas do pós-parto e o risco de trombose. Os POs podem estar relacionados com o desenvolvimento de Diabetes Mellitus tipo 2 na mulher que amamenta.

Segurança para a criança amamentada: Há evidência que não existe alteração consistente na produção do leite materno nem no peso ou crescimento do bebé, com o uso da $\mathrm{CH}$.

Conclusões: $\mathrm{O}$ achado de que as companhias farmacêuticas dirigem a maioria dos estudos sobre contraceptivos orais aumenta a preocupação acerca de potenciais viéses comerciais. São necessários estudos controlados e aleatorizados, de longa duração e de elevada qualidade, sobre a segurança dos COCs e POs no período pós-parto.

Palavras-chave: Contraceptivos Orais; Segurança; Período Pós-Parto.

\section{INTRODUÇÃO}

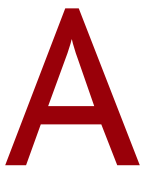
contracepção para a mulher no pós-parto é um verdadeiro problema de saúde pública. ${ }^{1}$ Em todo mundo e todos os anos, mais de cem milhões de mulheres tomam a decisão

\footnotetext{
* Interno de Medicina Geral e Familiar Centro de Saúde da Senhora da Hora - Unidade Local de Saúde de Matosinhos, EPE
}

de iniciar ou retomar a contracepção neste período da sua vida. ${ }^{1,2}$

Em Portugal, segundo o quarto Inquérito Nacional de Saúde, a pílula foi o método contraceptivo mais utilizado, e o Centro de Saúde foi o local de vigilância dos métodos contraceptivos mais frequentemente referidos pelas mulheres. ${ }^{3}$

Sabe-se que ao fim do primeiro mês após o parto cerca de $66 \%$ das mulheres a amamentar já retomaram 
a sua actividade sexual, e no final do segundo mês a percentagem sobe para cerca de $88 \%{ }^{4}$

Quando se avalia a segurança de um método contraceptivo é de suma importância definir o que se pretende medir. ${ }^{5,6}$ Os contraceptivos são um grupo farmacológico em que a segurança pode ser medida pelas reacções adversas graves e por outros parâmetros especificamente atribuídos a este grupo, como por exemplo o da reversibilidade do próprio método em termos de fertilidade para a mulher. ${ }^{6}$

O uso dos Contraceptivos Orais Combinados (COCs) e dos Progestativos Orais (POs) tem levantado questões importantes quanto à sua segurança quer para a mulher no pós-parto, quer para o seu bebé que é amamentado. Os receios surgem principalmente por estas hormonas poderem condicionar risco trombogénico acrescido para a puérpera, e causar alterações quantitativas e qualitativas no leite materno, pondo em causa o desenvolvimento e a saúde do bebé amamentado. Infelizmente estas dúvidas muitas vezes têm como resposta decisões baseadas em informação obsoleta, errada e

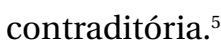

Por tudo isto, é importante para o Médico de Família adequar o método contraceptivo à mulher no pós-parto, promovendo uma vivência da sexualidade de forma saudável e segura, baseando-se na melhor evidência científica disponível. ${ }^{7,8}$

Este estudo tem como objectivo determinar a segurança dos COCs e dos POs para a mulher no período pós-parto e para a criança que é amamentada.

\section{MÉTODOS}

Foi realizada uma pesquisa nas bases de dados $M E D L I-$ NE, Guidelines Finder, National Guideline Clearinghouse, Canadian Medical Association Infobase, The Cochrane Library, DARE, Bandolier, TRIP e InfoPoems, de revisões baseadas na evidência, normas de orientação clínica, meta-análises, revisões sistemáticas e ensaios clínicos controlados e aleatorizados, publicados desde 1 de Janeiro de 2000 até 30 de Abril de 2008, em Inglês, Português, Francês e Castelhano, utilizando os termos MeSH: Contraceptives, Oral; Safety; Postpartum Period; e o termo Postpartum. Foram também pesquisadas recomendações de Sociedades Científicas, tendo sido seleccionadas apenas aquelas em que foi analisado o nível de evidência.
Os critérios utilizados para a inclusão dos artigos nesta revisão foram:

- População: mulher no período pós-parto que toma COC ou PO e a criança amamentada.

- Intervenção: Toma diária de COC ou de PO pela mulher no período pós-parto.

- Comparação: COC versus PO.

- Resultados: Segurança para a mulher no período pós-parto (risco de eventos cardiovasculares nomeadamente tromboembólicos, morbilidade e mortalidade). Segurança para a criança amamentada (desenvolvimento estato-ponderal e psicomotor).

A pesquisa, efectuada entre 1 de Março de 2008 e 30 de Abril de 2008, foi inicialmente realizada com os termos MeSH: Contraceptives, Oral; Safety e Postpartum Period (que no dicionário MeSH define, nas mulheres, o período logo após o parto), obtendo 52 artigos. Destes, foram excluídos 48 artigos por se repetirem ou por haver discordância com o objectivo da revisão e por não cumprirem os critérios de inclusão previamente descritos. Obtiveram-se, assim, três normas de orientação clínica e uma meta-análise que foram incluídas na revisão. Posteriormente, foi substituído o termo Postpartum Period, pelo termo Postpartum, uma sugestão da base de dados MeSH (período temporal mais alargado). A busca foi alargada, obtendo-se 64 artigos. Destes, 46 artigos foram excluídos por já terem sido encontrados na busca anterior e 12 artigos foram excluídos por se repetirem ou por discordância com o objectivo da revisão. Incluíram-se, assim, nesta segunda busca uma meta-análise, uma revisão sistemática, duas revisões baseadas na evidência e duas normas de orientação clínica.

No Índex de Revistas Médicas Portuguesas, a pesquisa foi feita com a combinação dos termos da lista dos Descritores em Ciências da Saúde (DeCS), usando os descritores MeSH portugueses: Contraceptivos Orais; Segurança e Período Pós-Parto, não se obtendo nenhum artigo.

Assim sendo, duas meta-análises, uma revisão sistemática, duas revisões baseadas na evidência e cinco normas de orientação clínica foram incluídas nesta revisão.

Para a comparação dos resultados de duas das normas de orientação clínica obtidas, foram usadas as categorias para o uso de contracepção hormonal segun- 
do os Critérios de Elegibilidade Médica da Organização Mundial de Saúde (CEMOMS): Categoria 1 - condição na qual não há restrição para o uso do método contraceptivo; Categoria 2 - condição onde as vantagens do uso do método contraceptivo na generalidade se sobrepõe aos riscos teóricos ou provados; Categoria 3 - condição onde os riscos teóricos ou provados na generalidade se sobrepõe às vantagens do uso do método contraceptivo e Categoria 4 - condição que representa um risco de saúde inaceitável se o método contraceptivo for usado. ${ }^{9,10}$

Para avaliar a qualidade dos estudos e a força de recomendação, foi utilizada a escala de Strength of Recommendation Taxonomy (SORT) da American Family Physician (AFP). ${ }^{11}$ Segundo esta taxonomia, a qualidade do estudo está subdividida em três Níveis de Evidência (Nível de Evidência 1: estudos de boa qualidade, evidência orientada para o doente; Nível de Evidência 2: estudos de qualidade limitada, evidência orientada para o doente; e Nível de Evidência 3: outra evidência) e a Força de Recomendação em três graus (Força de Recomendação A: consistente, boa qualidade, evidência orientada para o doente; Força de Recomendação B: inconsistente ou qualidade limitada, evidência orientada para o doente; e Força de Recomendação C: consensos, evidência orientada para a doença). ${ }^{11}$

\section{CORPO DE REVISÃO}

Os resultados foram agrupados em segurança para a mulher no período pós-parto e segurança para a criança que é amamentada. Posteriormente procede-se à discussão dos mesmos.

\section{RESULTADOS}

\section{Segurança para a mulher no período pós-parto}

Os COCs, quando comparados com os POs, no que diz respeito ao risco trombogénico, são, segundo as últimas normas de orientação clínica dos CEMOMS e dos Critérios de Elegibilidade Médica do Reino Unido (CEMRU), teoricamente menos seguros durante as primeiras três semanas pós-parto. Depois deste período, o risco trombogénico diminui, com a normalização da coagulação sanguínea e fibrinólise. Ainda, segundo estas normas de orientação clínica, baixas doses de POs não estão associadas com o risco trombótico, recomendando o seu uso em qualquer altura do pós-parto (Quadro I).9,10

Uma revisão de Junho de 2007 apresentada pelo $\mathrm{Eu}$ ropean Journal of Contraceptive and Reproductive Health Care, afirma que é pouco conhecido o risco de tromboembolismo venoso dos POs. ${ }^{12}$

Num grande estudo de coorte em mulheres latinas que tinham tido Diabetes Mellitus gestacional, no pós-parto, aquelas que estavam a amamentar e nas quais se

QUADRO I. Critérios para iniciar contraceptivos orais no período pós-parto segundo os CEMOMS e os CEMRU

\begin{tabular}{|c|c|c|c|c|c|}
\hline & & \multicolumn{2}{|c|}{ Contraceptivo Oral Combinado } & \multicolumn{2}{|c|}{ Progestativo Oral } \\
\hline & & CEMOMS & CEMRU & CEMOMS & CEMRU \\
\hline \multirow{2}{*}{ Não amamenta } & $<21$ dias & 3 & 3 & 1 & 1 \\
\hline & $\geq 21$ dias & 1 & 1 & 1 & 1 \\
\hline \multirow{4}{*}{ Amamenta } & $<6$ semanas & 4 & 4 & 3 & 1 \\
\hline & $\begin{array}{l}\geq 6 \text { semanas }<6 \text { meses } \\
\text { (amamentação exclusiva) }\end{array}$ & 3 & 3 & 1 & 1 \\
\hline & $\begin{array}{l}\geq 6 \text { semanas }<6 \text { meses } \\
\text { (amamentação parcial) }\end{array}$ & & 2 & & 1 \\
\hline & $\geq 6$ meses & 2 & 1 & 1 & 1 \\
\hline
\end{tabular}

CEMOMS = Critérios de Elegibilidade Médica da Organização Mundial de Saúde

CEMRU = Critérios de Elegibilidade Médica do Reino Unido

Categorias:

Categoria 1 - condição na qual não há restrição para o uso do método contraceptivo;

Categoria 2 - condição onde as vantagens do uso do método contraceptivo na generalidade se sobrepõe aos riscos teóricos ou provados;

Categoria 3 - condição onde os riscos teóricos ou provados na generalidade se sobrepõe às vantagens do uso do método contraceptivo;

Categoria 4 - condição que representa um risco de saúde inaceitável se o método contraceptivo for usado. 
prescreveram POs, verificou-se um aumento na proporção de desenvolvimento de Diabetes Mellitus do tipo 2 durante os primeiros dois anos comparando com aquelas às quais eram administradas doses baixas de COCs. $\mathrm{O}$ risco de desenvolvimento de Diabetes Mellitus do tipo 2 aumentou com a duração da exposição ao POs. ${ }^{13}$

\section{Segurança para a criança que é amamentada}

Nas últimas normas de orientação clínica dos CEMOMS e CEMRU, os COCs são contra-indicados na mulher que amamenta durante as primeiras seis semanas. Esta contra-indicação segundo as mesmas orientações clínicas deve-se ao facto de haver um risco teórico de os estrogéneos administrados à mulher poderem provocar alterações no desenvolvimento do fígado e cérebro dos recém-nascidos a amamentar. Estas orientações clínicas referem também que, das seis semanas até aos seis meses da criança, há evidência limitada no que concerne aos riscos do uso de COCs durante a amamentação. Referem potencial diminuição da quantidade do leite e diminuição da duração da lactação, podendo afectar o crescimento da criança. ${ }^{8-10}$ As normas de orientação clínica da Faculty of Family Planning and Reproductive Health Care Clinical Effectiveness Unit (FFPRHC) citadas nos CEMRU, sugerem que os COCs apenas devem ser usados a partir das seis semanas do pós-parto, numa amamentação estabelecida, se outro método não for possível. ${ }^{14,15}$ Os CEMRU fazem também a distinção entre amamentação exclusiva e amamentação parcial. ${ }^{10}$ Após os seis meses pós-parto, o risco para a criança diminui segundo as duas normas. ${ }^{9,10}$

Quanto ao uso dos POs antes das seis semanas pós-parto, os CEMOMS referem preocupação no seu uso, devido a prováveis alterações no leite materno ou no crescimento do bebé. Quer os CEMOMS, quer os CEM$\mathrm{RU}$, referem que os POs podem ser usados com segurança após as seis semanas do pós-parto.,10

Segundo o Colégio Americano de Obstetras e Ginecologistas (ACOG), o uso de Contracepção Hormonal Combinada (CHC) pelas mulheres que amamentam não deve ser iniciado antes das seis semanas do pós-parto. Após as seis semanas e nas mulheres que apresentam um bom estado nutricional, o seu uso parece não resultar em problemas de desenvolvimento para a criança, sendo a sua utilização aconselhada apenas quando a lactação estiver bem estabelecida. ${ }^{16}$
Uma revisão da Cochrane, publicada em 2003, utilizada como referência nos artigos supracitados, analisou cinco ensaios clínicos, comparando a CHC com a contracepção não hormonal e com a contracepção com apenas progestagénio na lactação, usando como critérios de inclusão a contracepção oral ou injectável. Esta revisão concluiu que os ensaios clínicos foram insuficientes para estabelecer qualquer efeito da $\mathrm{CH}$ na qualidade e quantidade do leite e no crescimento da criança. ${ }^{1}$

Uma revisão baseada na evidência, publicada na revista American Family Physician, refere não ter sido verificada alteração consistente na produção do leite materno nem no peso ou crescimento da criança com uso de COCs (Força de Recomendação A). ${ }^{17}$

\section{DISCUSSÃO}

\section{Segurança para a mulher no período pós-parto}

No que concerne aos resultados obtidos quanto à segurança dos COCs e POs para a mulher no pós-parto, é de referir que os artigos encontrados focam a sua atenção no potencial risco trombogénico. Verificou-se a inexistência de estudos aleatorizados, controlados e de boa qualidade que comprovassem o real efeito prótrombótico com uso de COCs. A única informação disponível é baseada em risco teórico e orientada para a doença. ${ }^{8,9,10}$ Quanto ao uso dos POs e o risco trombótico existem estudos de qualidade limitada e inconsistente, mas orientados para o doente. ., $, 9,10,12^{2}$ Conclui-se, assim, que os COCs são menos seguros durante as primeiras três semanas pós-parto (Nível de Evidência 3). Doses baixas de POs não estão associadas com o risco trombótico, recomendando o seu uso em qualquer altura do pós-parto (Nível de Evidência 2).

Nesta revisão foi encontrado apenas mais um risco adicional para a mulher, além do risco trombogénico: o risco de desenvolver Diabetes Mellitus do tipo 2, associado ao consumo de POs, em mulheres que tinham tido Diabetes Mellitus gestacional (Nível de Evidência 3).$^{13}$ Contudo, este achado deve ser ainda confirmado em estudos que contenham mulheres previamente saudáveis, de todas as raças e que estejam ou não a amamentar, para que se possam generalizar os dados para as mulheres no puerpério.

\section{Segurança para a criança que é amamentada}

Quanto à segurança para a criança amamentada foi in- 
cluída a norma de orientação clínica do ACOG. ${ }^{16}$ Nela é referido que o uso de $\mathrm{CHC}$ depois das seis semanas pós-parto e nas mulheres que apresentam um bom estado nutricional parece não resultar em problemas de desenvolvimento para a criança, aconselhando o seu uso apenas quando a lactação estiver bem estabelecida (Força de Recomendação B) ${ }^{16}$

Foram também incluídos os resultados analisados por duas grandes sociedades científicas mundiais que desenvolveram os Critérios de Elegibilidade Médica para o uso de contraceptivos; os CEMOMS e CEMRU.,10 Ambas se basearam em riscos teóricos, em estudos aleatorizados e controlados mas de qualidade limitada, e na única revisão sistemática da Cochrane acerca deste assunto incluída neste estudo. Essa revisão da Cochrane analisou cinco ensaios clínicos de fraca qualidade e com evidência discordante. ${ }^{1}$ Esses ensaios clínicos foram insuficientes para estabelecer qualquer efeito da $\mathrm{CH}$ na qualidade e quantidade do leite materno, e no crescimento das crianças amamentadas. ${ }^{1}$ A revisão baseada na evidência encontrada, relativa a este tópico, citando a mesma revisão da Cochrane, refere evidência de boa qualidade, no que concerne a não ser verificada alteração consistente na produção do leite materno nem no peso ou crescimento do bebé com uso de COCs (Força de Recomendação A). ${ }^{17}$

É importante realçar que grande parte dos artigos obtidos na pesquisa, foi produzida por companhias farmacêuticas, aumentando a preocupação acerca de potenciais viéses comerciais. ${ }^{5}$ Estes viéses fazem-se sentir sobretudo no incentivo à prescrição. Contudo, como nenhum desses estudos cumpriu satisfatoriamente os critérios de inclusão, foram automaticamente excluídos. Por esse motivo, neste estudo, esses viéses foram praticamente eliminados.

\section{CONCLUSÃO}

A contracepção no pós-parto é uma questão essencial que se coloca à futura mãe, e à qual o seu Médico de Família deve dar resposta, baseando-se na melhor evidência.

O clínico deve adequar um método contraceptivo seguro às necessidades e desejos da mulher para a contracepção no pós-parto. A escolha e o início do método contraceptivo é uma decisão importante para a mulher que amamenta, devendo esta questão ser oportu- namente discutida com a puérpera.

Da evidência actualmente disponível os COCs são menos seguros durante as primeiras três semanas pós-parto (Força de Recomendação C).

Doses baixas de POs não estão associadas com o risco trombótico, recomendando o seu uso em qualquer altura do pós-parto (Força de Recomendação B).

Não foi provada alteração consistente na produção do leite materno nem no peso ou crescimento da criança que é amamentada, e cuja mãe toma $\mathrm{CH}$, especialmente COC (Força de Recomendação A). ${ }^{17}$

São necessários mais estudos controlados, aleatorizados, de longa duração e de boa qualidade que contenham todos os pressupostos, incluindo os éticos, sobre a segurança dos COCs e POs, orientados para a mulher no pós-parto que deseja contracepção e para a criança amamentada. Estes estudos deverão esclarecer dúvidas levantadas nesta revisão, nomeadamente: o verdadeiro risco trombogénico para a puérpera com o uso da $\mathrm{CH}$ oral, o provável aparecimento de Diabetes Mellitus do tipo 2 na puérpera que toma POs e o risco no desenvolvimento estato-ponderal e psicomotor da criança que é amamentada.

\section{REFERÊNCIAS BIBLIOGRÁFICAS}

1. Truitt ST, Fraser AB, Grimes DA, Gallo MF, Schulz KF. Combined hormonal versus nonhormonal versus progestin-only contraception in lactation. Cochrane Database Syst Rev 2003; (2): CD003988.

2. King J. Contraception and lactation. J Midwifery Womens Health 2007 Nov-Dec; 52 (6): 614-20.

3. Instituto Nacional de Estatística. $4^{\circ}$ Inquérito Nacional de Saúde 2005/2006. INE; 2007. Disponível em: http://www.ine.pt/xpor$\mathrm{tal} / \mathrm{xmain}$ ?xpid=INE\&xpgid=ine_destaques\&DESTAQUESdest_boui $=6449945 \& D E S T A Q U E S m o d o=2 \& x l a n g=p t$ [acedido em 20/04/2008].

4. Godinho C, Lopes J. Contracepção no pós-parto. Arq Mat Alfredo da Costa 2001; 17 (2): 111-5.

5. Helmerhorst FM, Belfield T, Kulier R, Maitra N, O'Brien P, Grimes DA. The Cochrane Fertility Regulation Group: synthesizing the best evidence about family planning. Contraception. 2006 Oct; 74 (4): 280-6.

6. Kapp N, Curtis KM, Borgatta L. Study design to evaluate the safety and effectiveness of hormonal contraception for women. Clin Obst Gynecol 2007 Dec; 50 (4): 850-67.

7. Direcção-Geral da Saúde, Divisão de Saúde Materna, Infantil e dos Adolescentes. Saúde reprodutiva - Planeamento familiar. Orientações técnicas. Ed. rev. actualizada. Lisboa: DGS; 2001.

8. Lesnewski R, Prine L. Initiating hormonal contraception. Am Fam Physician 2006 Jul 1; 74 (1): 105-12.

9. World Health Organization. Medical Eligibility Criteria for Contraceptive Use. 3rd ed. Geneva:WHO; 2004. Disponível em: http://www.who. 
int/reproductive-health/publications/mec/mec.pdf [acedido em 04/04/2008].

10. UK Medical Eligibility Criteria for Contraceptive Use (2005/2006). Faculty of Family Planning and Reproductive Health Care Clinical Effectiveness Unit; 2006. Disponível em: http://www.fsrh.org/admin/uploads/298_UKMEC_200506.pdf [acedido em 04/04/2008].

11. Ebell MH, Siwek J, Weiss BD, Woolf SH, Susman J, Ewingman B, et al. Strength of Recommendation Taxonomy (SORT): A patient-centered approach to grading evidence in the medical literature. Am Fam Physician 2004 Feb 1; 69 (3): 548-56.

12. Martínez F, Avecilla A. Combined hormonal contraception and venous thromboembolism. Eur J Contracept Reprod Health Care 2007 Jun; 12 (2): 97-106

13. Damm P, Mathiesen ER, Petersen KR, Kjos S. Contraception after gestational diabetes. Diabetes Care 2007 Jul; 30 Suppl 2: S236-41.

14. Faculty of Family Planning and Reproductive Health Care. FFPRHC Guidance (July 2004): Contraceptive choices for breastfeeding women. J Fam Plann Reprod Health Care 2004 Jul; 30 (3): 181-9.

15. Faculty of Family Planning and Reproductive Health. FFPRHC Guidance (July 2006): First Prescription of Combined Oral Contraception. Disponível em: http://www.ffprhc.org.uk/admin/uploads/FirstPrescCombOralCont]an06.pdf [acedido em 06/04/2008].
16. American College of Obstetricians and Gynecologists (ACOG) Committee on Practice Bulletins-Gynecology. ACOG practice bulletin. No. 73: Use of hormonal contraception in women with coexisting medical conditions. Obstet Gynecol 2006 Jun; 107 (6): 1453-72.

17. Roederer MW, Blackwell JC. FPIN's clinical inquiries: risks and benefits of combination contraceptives. Am Fam Physician 2006 Dec 1; 74 (11): 1915-6.

\section{AGRADECIMENTOS}

À Dr. ${ }^{a}$ Raquel Braga, minha orientadora de formação, pela colaboração na orientação metodológica desta revisão.

\author{
ENDEREÇO PARA CORRESPONDÊNCIA \\ Luís Filipe Cavadas \\ Centro de Saúde da Senhora da Hora \\ Rua da Lagoa, $s / n^{\circ}$ \\ 4460 Senhora da Hora \\ Telef.: 967241978 \\ E-mail: luisfilipemcavadas@gmail.com
}

Recebido em 23/06/08

Aceite para publicação em 12/12/08

\section{ABSTRACT}

Introduction: Hormonal Contraception (HC) after childbirth is a public health issue of global importance, with repercussion for both mother and child.

Objectives: To determine the safety of Combined Oral Contraceptives (COCs) and Progestogen-only pills (POPs) in postpartum women and breastfed children.

Methods: The author searched MEDLINE, Guidelines Finder, National Guideline Clearinghouse, Canadian Medical Association, Infobase, The Cochrane Library, DARE, Bandolier, TRIP, InfoPoems and Scientific Societies, using the MeSH terms: Contraceptives, Oral; Safety; Postpartum Period; and the term Postpartum. The search was limited to articles published between January 2000 and April 2008 in English, Portuguese, French and Spanish. The categories of the World Health Organization Medical Eligibility Criteria for Contraceptive Use (WHOMEC) were used to compare results. The Strength of Recommendation Taxonomy (SORT) of the American Family Physician was used to assess the Level of Evidence.

Results: Two meta-analysis, one systematic review, two evidence based reviews and five guidelines met the inclusion criteria. Safety for Women: there is theoretical concern regarding the association between COCs use up to three weeks postpartum and the risk of thrombosis. POPs can be related with the development of Type 2 Diabetes Mellitus in breastfeeding Women. Safety for breastfed children: there is evidence that there is no consistent change in breast milk production, child growth or weight, with the use of HC.

Conclusion: The finding that pharmaceutical companies conducted most of the trials on oral contraceptives raises concerns about potential commercial biases. It would be advisable to do high quality, long-term, controlled and randomised studies about the safety of COCs and POPs in the postpartum period.

Keywords: Contraceptives; Oral; Safety; Postpartum Period. 